\title{
Efficacy of Corticosteroids in Acute Respiratory Distress Syn- drome: An Observational Study
}

\author{
Prabhu Varsha A', Awasthi Shivanshu', Shanavas Rithu', Kunhikatta Vijayanarayana', Thunga Girish', Nair Sreedharan', Acharya Raviraja ${ }^{2}$ \\ 'Department of Pharmacy Practice, Manipal College of Pharmaceutical Sciences, Manipal University, Manipal, INDIA. \\ 2Department of General Medicine, Kasturba Medical College, Manipal, INDIA.
}

\begin{abstract}
Background: Deficits in the treatment options makes Acute Respiratory Distress Syndrome (ARDS) management more challenging. With Involvement of inflammatory pathways, corticosteroid remains a vital choice for ARDS treatment. However lack of studies and presence of ambiguity in results with use of glucocorticoids makes it imperative to carry out more research. The aim of the study was to assess the efficacy of glucocorticoids in the treatment of ARDS and to identify the treatment pattern of ARDS. Methods: Retrospective observational study, involving 150 patients with confirmed diagnosis of ARDS admitted during January 2011 to December 2011 in a tertiary care hospital. Demographics, social habits, and association between glucocorticoid initiation and respective onset and outcomes like mortality and free ventilator days, were analysed by Chi square test and $p<0.05$ was considered to be statistically significant. Data analysis was done by using SPSS version 16. Results: Mean age of our study population was $43.37 \pm 16.15$ with male $60.7 \%$ and $39.3 \%$ female. The study found reduced mortality $(41.7 \%)$ in the glucocorticoid treatment group compared to the non-glucocorticoid group (56.4\%) $(p<0.022)$. Among the glucocorticoid treated subgroups, in the early start subgroup there was a statistically significant reduction of mean ventilator days $(4.61 \pm 2.81$ vs $6.44 \pm 4.47)$ and mortality $(30.9 \%$ vs $80 \%, p<0.001)$. Conclusions: The use of glucocorticoids in ARDS showed benefits, especially when started early in the course of
\end{abstract}

treatment with reduced mortality and decreased ventilator days. However small sample size precluded the power of detection of important outcome such as mortality and more research is necessary to establish the role of glucocorticoid therapy in ARDS.

Key words: ARDS, Glucocorticoids, Mortality.

Key messages: Glucocorticoids used in the treatment of ARDS might be beneficial in terms of reduced mortality and decreased ventilator days. This was purely an observational study so, irrespective of what was found, the quality of the conclusions does not solves this long and interesting dispute.

Correspondence :

Dr. Vijayanarayana Kunhikatta,

Department of Pharmacy Practice, Manipal College of Pharmaceutical Sciences, Manipal University, Manipal-576104, INDIA.

Email: vijayanarayana.k@manipal.edu

DOI: 10.5530/jyp.2017.9.13

\section{INTRODUCTION}

Acute respiratory distress syndrome (ARDS) is an acute hypoxemic respiratory failure due to systemic or pulmonary insults and is the most severe form of acute diffuse inflammatory lung injury, characterized by diffuse alveolar damage, alveolar capillary leakage and protein rich pulmonary edema. ${ }^{1,2}$ There are many etiological factors contributing to ARDS. However, only a few account for majority of the cases like sepsis from both pulmonary and non-pulmonary origin. ${ }^{3}$ Other common etiologies for ARDS includes pneumonia, aspiration of gastric contents, severe trauma with shock, multiple transfusions, near drowning and fat emboli. ${ }^{4}$ Mortality in ARDS lies in between $30-40 \%{ }^{5}$

Patients with ARDS from direct lung injury like pneumonia, aspiration and pulmonary contusion have twice the mortality of those with indirect cause of lung injury such as major trauma, multiple blood transfusions. ${ }^{6}$ The diagnosis of ARDS is done based on three main criteria's: the presence of acute severe hypoxemia, bilateral infiltrates on chest radiography (CXR), and absence of raised pulmonary artery wedge pressure. ${ }^{1}$ The pathophysiology of ARDS, the most important feature is increased capillary permeability. This is due to damage of the capillary endothelium and alveolar epithelium lining causing accumulation of protein rich fluid inside the alveoli. The subsequent alveolar damages lead to the release of pro-inflammatory cytokines, such as Tumor Necrosis Factor (TNF), Interleukin (IL) 1 and 6. These cytokines recruit the neutrophils to the site of damage. On activation neutrophils release toxic mediators such as reactive oxygen species and proteases leading to oxidative cell damage. Other factors like endothelin-1, angiotensin-2 and phospholipase A-2 can enhance vascular permeability and micro-vascular architecture which in addition can aggravate inflammation leading to lung damage.

After fluid accumulation, proliferation phase occurs which is characterized by pulmonary edema, proliferation of type 2 alveolar cells, fibroblasts, myofibroblasts and new matrix deposition. During this phase patients exhibit impairment in pulmonary compliance, severe hypoxia, ventilator dependence and higher mortality. ${ }^{8}$ The pulmonary hypertension associated with ARDS can cause parenchymal damage, airway collapse, hypoxic pulmonary vasoconstriction, presence of other pulmonary vasoconstrictors and vascular compression. ${ }^{9}$ ARDS can be categorized an early ( 1 to 7 days after onset), early persistent (7 to 14 days) \& late persistent ( $>14$ days) which can also be considered as fibroproliferative or unresolving ARDS. ${ }^{10,11}$

In ARDS the primary therapeutic goal is to treat the underlying cause of the disease along with supportive therapy. It includes infection control, volume resuscitation, thromboprophylaxis combined with focused ventilator strategies with appropriate treatment of the underlying causes. There are no effective pharmacological therapies for ARDS.

While inflammation is one of the vital pathways in the pathogenesis of ARDS with the involvement of inflammatory mediators, ${ }^{1}$ glucocorticoids could play a crucial role in precluding it. How-

This is an open access article distributed under the terms of the Creative Commons Attribution-NonCommercial-ShareAlike 4.0 License, which allows others to remix, tweak, and build upon the work non-commercially, as long as the author is credited and the new creations are licensed under the identical terms. 
Prabhu V et al.: Corticosteroids role in Acute Respiratory Distress Syndrome.

ever, the role and efficacy of the glucocorticoids in the treatment of ARDS is still controversial and yet to be established. Insufficient data and lack of the studies to demonstrate the use of glucocorticoids in ARDS makes it imperative to carry out more research in this area. ${ }^{20}$

\section{Objective}

The aim of the study was to assess the efficacy of glucocorticoids in the treatment of ARDS and to identify the treatment approach of ARDS

\section{MATERIALS AND METHODS}

This was a retrospective observational study conducted in a tertiary care hospital in Southern region of Karnataka. All patients diagnosed with ARDS and confirmed by chest X-ray and arterial blood gas analysis, during January 2011 and December 2011 were identified from Medical Records Division (MRD) registry using ICD code J80. Ethical approval was obtained from the institutional ethics committee prior to initiation of the study. Data was collected from 150 patients with ARDS. Patients with co-morbid illnesses like bronchial asthma and chronic obstructive pulmonary disease (COPD), immunocompromised patients including $\mathrm{Hu}$ man Immunodeficiency Virus (HIV) positive, history of bone marrow or solid organ transplantation, current malignancy, neutropenia, receiving cytotoxic therapy for any reason and acute burn injury were excluded from the study.

A case record form (CRF) was developed and validated by local experts for its appropriateness for the collection of data. Patients demographical details like name, age, sex, month of admission, medical and medication history, time of onset of ARDS, symptoms of patients during the admission, vital signs, reports of laboratory investigations, pulse oximetry readings along with the duration of ventilator days, duration of hospitalization days and treatment chart including dose and time of start of glucocorticoids were recorded in the CRF. The study mainly emphasized the decision and timing of glucocorticoid treatment on ARDS patients. To analyze the association between glucocorticoid therapy and efficacy, patients were categorized as survived and expired.

Outcome analysis investigated efficacy of glucocorticoids and the survival rate of patients along with the timing of glucocorticoid therapy initiation, categorized as early onset $(<72 \mathrm{~h})$ and late onset (after $72 \mathrm{~h})$.

\section{Statistical Analysis}

Demographics, social habits, and associations between glucocorticoid initiation and respective onset and outcomes like mortality and free ventilator days, were analysed by Chi square test and $\mathrm{p}<0.05$ was considered statistically significant. Data analysis was done by using SPSS version 16.

\section{RESULTS}

During the study period, a total of 150 patients diagnosed with ARDS and admitted to medicine wards of the hospital were enrolled in the study, based on the inclusion and exclusion criteria. Demographic details of the patients are given in the Table 1. Mean age of the study population was $43.37 \pm 16.15$ years. Among the study population $22.6 \%$ were smokers and $19.8 \%$ were alcoholics. Month-wise wise admission rate analysis showed highest rate of incidence during July to September (14-22\%) and lowest during October to December. Rate of incidence were variable during January to June. Among the study population Sepsis (32\%) and Pneumonia (21.3\%) were the most common infective etiological factors for ARDS while post operative ARDS (15\%) was the non-infectious etiological factor.

Clinical characteristics of ARDS are mentioned in the Table 2. Breathlessness (52\%), fever (79.3\%), fatigue (12\%), cough (26\%) and edema (6\%) were the common symptoms at presentation. Outcomes of the patients enrolled $(\mathrm{n}=150)$ in the study were analyzed. Among the study population $54.7 \%$ patients recovered from ARDS while the mortality rate was $45.3 \%$.

Treatment pattern analysis revealed that $98.6 \%$ of patients with ARDS were given antibiotics for their underlying conditions and that $48.6 \%$ of patients received glucocorticoids. However supportive treatment remained mainstay for ARDS treatment and it included mechanical ventilation, oxygenation, bronchodilators, vasopressor agents, volume resuscitation, and sodium bicarbonate. Common treatment options used in ARDS are described in Table 3.

Study population was categorized into two main groups based on the glucocorticoid therapy decision, demarking a glucocorticoid treated group and a non-glucocorticoid treated group (Table 4). Higher mortality rate was observed in the non-glucocorticoid treated group (56.4\%) compared to the glucocorticoid treated patients $(41.7 \%)$, being this difference statistically significant $(\mathrm{p}<0.022)$.

Study population was further categorized in two subgroups based on the time of start of the glucocorticoid therapy (details in Table 5). One subgroup received early glucocorticoid therapy $(<72 \mathrm{~h})$, whereas other subgroup received late glucocorticoid therapy $(>72 \mathrm{~h})$. Patient who received late glucocorticoid therapy had higher mortality rate $(80 \%)$. The group of patients that received early glucocorticoid therapy showed a significant higher recovery rate $(69.1 \%)(\mathrm{p}<0.001)$.

Regarding the timing effect of glucocorticoid therapy on ventilator days (Table 6), the subgroup of patients who received early glucocorticoid therapy had less mean ventilation days $(4.61 \pm 2.81)$ compared to the subgroup who received late glucocorticoid therapy $(6.44 \pm 4.47)$

\section{DISCUSSION}

This was a retrospective observational study conducted in a tertiary care hospital. The primary objective was to assess the role of glucocorticoid in all stages of ARDS and to explore the treatment pattern used in the management of ARDS. A total of 150 ARDS patients data was collected and analyzed. Gender distribution had a male to female ratio of $2: 1$. This was similar to the study conducted by Hemptinne et al, in which $59 \%$ of ARDS patients were male. ${ }^{13}$ Social habits of study population revealed that $19.8 \%$ patients were alcoholic and $22.6 \%$ were smokers. A study conducted by Iribarren C et al., showed that cigarette smoking was associated with $50 \%$ of the ARDS cases; however alcohol consumption had insignificant association with ARDS occurrence. Pathologically smoking may cause alveolar damage which could eventually leads to respiratory insufficiency and ARDS. Cigarette contains high amount of reactive oxygen species which can result in membrane peroxidation and itcan worsen theinflammation. ${ }^{14}$ Incidence of ARDS was shown to be higher during the months of July to September and this period is considered as monsoon season in the region of India where this study was conducted. A study conducted by Bajpai et al, also shows that monsoon related illnesses account for higher incidence of ARDS in September. ${ }^{15}$ Sepsis (32\%), pneumonia (21.3\%) and scrub typhus (20\%) were the primary etiological causes for ARDS in our study population. A study conducted by Lu et al also reported sepsis (30.6\%) as the major etiological cause of ARDS. ${ }^{16}$

There was higher mortality rate in the group of patients who did not receive glucocorticoids (56.4\%) compared to those who received glucocorticoid therapy $(41.7 \%)$. The result was fairly consistent with a large scale trial conducted by ARDSnet which showed the higher mortality rate in the methylprednisolone treated group (27\%) compared to placebo group (36\%). ${ }^{18}$ Another trial conducted by Meduri GU et al., also showed lower mortality rate in the early onset low dose methylpredniso- 
Prabhu V et al.: Corticosteroids role in Acute Respiratory Distress Syndrome.

Table 1: Patient Demographic details

\begin{tabular}{cc}
\hline Patient Demographic details & $\mathbf{N}=150$ \\
\hline Mean Age \pm SD in years & $43.37 \pm 16.15$ \\
Gender- wise Distribution: & $91(60.7 \%)$ \\
Male, $\mathrm{n}(\%)$ & $59(39.3 \%)$ \\
Female, n (\%) & \\
Social Habits & $20(22.6 \%)$ \\
Smoker & $18(19.8 \%)$ \\
Alcoholic & \\
Month of admission & $21(14 \%)$ \\
July & $28(18 \%)$ \\
August & $33(22 \%)$ \\
September & \\
Etiology & $10(15 \%)$ \\
Post operative & $48(32 \%)$ \\
Sepsis & $32(21.3 \%)$ \\
Pneumonia & $30(20 \%)$ \\
Scrub typhus & $11(7.3 \%)$ \\
Leptospirosis & $23(15.3 \%)$ \\
Others &
\end{tabular}

Table 2: Clinical features and outcomes

\begin{tabular}{cc}
\hline Symptoms & Frequency (\%) \\
\hline Breathlessness & $78(52 \%)$ \\
Fever & $119(79.3 \%)$ \\
Fatigue & $18(12 \%)$ \\
Cough & $39(26 \%)$ \\
Edema & $9(6 \%)$ \\
& \\
Outcome & Frequency (\%) \\
& \\
Recovered & $82(54.7 \%)$ \\
Death & $68(45.3 \%)$ \\
\hline
\end{tabular}

Table 3: Treatment pattern of ARDS

\begin{tabular}{cc}
\hline Treatment & Frequency (\%) \\
\hline Antibiotics & $148(98.6 \%)$ \\
Glucocorticoids & $73(48.6 \%)$ \\
Ventilation & $134(89.3 \%)$ \\
Oxygenation & $106(70.7 \%)$ \\
Bronchodilators & $43(28.7 \%)$ \\
Vasopressor agents & $84(56.0 \%)$ \\
Volume resuscitation & $131(87.3 \%)$ \\
Sodium bicarbonate & $9(6 \%)$ \\
\hline
\end{tabular}

Table 4: Comparison between glucocorticoid therapy and patient outcome

\begin{tabular}{ccc}
\hline Type of therapy & \multicolumn{2}{c}{ Frequency, $\mathbf{n}(\%)$} \\
\hline & Recovered & Expired \\
\cline { 2 - 3 } With glucocorticoid therapy & $42(58.3 \%)^{*}$ & $30(41.7 \%)$ \\
Without glucocorticoid therapy & $34(43.6 \%)$ & $44(56.4 \%)$ \\
\hline
\end{tabular}

Table 5: Time of start of glucocorticoid therapy and patient outcome

\begin{tabular}{ccc}
\hline $\begin{array}{c}\text { Time of start of Glucocorticoid } \\
\text { Therapy }\end{array}$ & \multicolumn{2}{c}{ Frequency, $\mathrm{n}(\%)$} \\
\hline & Recovered & Expired \\
Early start $(<72 \mathrm{Hr})$ & $38(69.1 \%)$ & $17(30.9 \%)$ \\
Late start $(>72 \mathrm{Hr})$ & $3(20 \%)$ & $12(80 \%)$ \\
\hline
\end{tabular}

Table 6: Comparison between time of start of glucocorticoid therapy and patient outcome

\begin{tabular}{cc}
\hline $\begin{array}{c}\text { Time of start of glucocorticoid } \\
\text { therapy }\end{array}$ & Frequency, $\mathrm{n}(\%)$ \\
\hline & Mean Ventilation days \\
\cline { 2 - 2 } Early start $(<72 \mathrm{Hr})$ & $4.61 \pm 2.81$ \\
Late start $(>72 \mathrm{Hr})$ & $6.44 \pm 4.47$ \\
\hline
\end{tabular}

lone treated group compared to placebo $(20.6 \% \pm$ standard deviation vs $42.9 \% \pm$ standard deviation). ${ }^{17}$

Considering recovery rate from ARDS, our study found that the early start of glucocorticoid therapy was more effective than a late start $(69.1 \%$ vs $20.0 \%$ ). A study conducted by Meduri GU et al., also showed that low dose glucocorticoid treatment started on an early stage significantly decreased ICU mortality (20.56\% vs. $42.9 \%) .{ }^{17}$ However, there is lack of uniform evidence regarding the place and timing of glucocorticoids in ARDS. Studies have reported controversial results such as the trial by ARDS clinical trial network (ARDSnet) concluded that early start glucocorticoid therapy showed no survival benefits. ${ }^{18}$

Nonetheless, there was a significant reduction in the mean ventilator days in the group of patients who received early start of therapy, compared to the late start $(4.61 \pm 2.81$ vs $6.44 \pm 4.47)$.

A large randomized controlled trial asserted the role of corticosteroids in early and late ARDS, with prolonged low-dose methylprednisolone. Although the study did not show mortality benefits from early start, it rendered rapid weaning from mechanical ventilation, reduced ventilator days and fast recovery, especially when methylprednisolone was initiated early in the treatment. ${ }^{20}$ Another study conducted by ARDS network and a meta-analysis by Peter JV et al., concluded that glucocorticoid therapy is beneficial in reducing mean ventilation days albeit there is a lack of comparison between early start and late start therapy and the bias effect derived from heterogeneous definitions of "early" and "late start" in available studies. ${ }^{18,19}$

\section{CONCLUSION}

Antibiotics along with vasopressor agents, volume resuscitation, bronchodilators, oxygenation, ventilation and sodium bicarbonate were the most commonly used supportive treatments. Study showed promising results in reversing the ARDS reaction and improving lung function (reduction in mean ventilator days) with the use of glucocorticoid therapy. Patients who received early glucorticoid therapy had a better recovery and less mortality, compared to those who received late glucocorticoid therapy ( $>72$ hours) in the course of disease. However power of detection of a critical outcome such as mortality is restrained by a small sample size, the retrospective nature of the study and by the non-uniformization of the glucocorticoid doses employed and it necessitates more research in order to establish a clear role of glucocorticoid in ARDS. 


\section{ACKNOWLEDGEMENTS}

We acknowledge the support given by Manipal University and Kasturba Hospital, Manipal.

\section{CONFLICT OF INTEREST}

No conflict of interest are declared.

\section{ABBREVIATIONS USED}

ARDS: Acute Respiratory Distress Syndrome; MRD: Medical Records Division; ICU: Intensive Care Unit; CRF: Case Record Form; COPD: Chronic Obstructive Pulmonary Diseases, ICD: International Classification of Diseases.

\section{REFERENCES}

1. Dushianthan A, Grocott M, Postle A, Cusack R. Acute respiratory distress syndrome and acute lung injury. Postgrad Med J. 2011;87(1031):612-22.

2. Chesnutt M, Gifford A, Prendergast T. Pulmonary disorders. In: McPhee J, Papadakis A, editors. Current medical diagnosis and treatment. $49^{\text {th }}$ ed. New Delhi: McGraw-Hill Medical; 2010. 291-3.

3. Rubenfeld GD, Caldwell E, Peabody E, Weaver J, Martin DP, Neff M et al. Incidence and outcomes of acute lung injury. N Engl J Med. 2005;353(16):1685-93.

4. Ware LB, Matthay MA. The acute respiratory distress syndrome. N Eng J Med 2000:342:1334-49.

5. Lamontagne F, Briel M, Guyatt GH, Cook DJ, Bhatnagar N, Meade M. Corticosteroid therapy for acute lung injury, acute respiratory distress syndrome, and Severe pneumonia:A meta-analysis of randomized controlled trials. Journal of Critical Care. 2010;25(3):420-35.

6. Levy BD, Shapiro SD. Acute Respiratory Distress Syndrome. In: Fauci AS, Braunwald E, Kasper D, Hauser SL, Longo DL, Jameson JL, Loscalzo J, editors. Harrison's Principles of Internal Medicine. 17th ed. New Delhi: McGraw-Hil
Medical; 2008.1680-84.

7. Windsor ACJ, Mullen PG, Fowler AA and Sugarman HJ. Role of neutrophil in adult respiratory distress syndrome. Br J Surg. 1993;80(1):10-17.

8. Martin C, Papazian L, Payan MJ, Saux P, Gouin F. Pulmonary fibrosis correlates with outcome in ARDS. A study in mechanically ventilated patients. Chest. 1995;107(1):196-200.

9. Zapol WM, Snider MT. Pulmonary hypertension in severe acute respiratory failure. N Engl J Med. 1977;296(9):476-80.

10. Curtis NS, Peter C Gay. Are Corticosteroids Useful in Late-Stage Acute Respiratory Distress Syndrome?. Respir Care. 2010;55(1):43-52.

11. Khilnani GC, Hadda V. Corticosteroids and ARDS: A review of treatment and prevention evidence. Lung India. 2011;28(2):114-9.

12. Meduri GU et al. Steroid treatment in ARDS: a critical appraisal of the ARDS network trial and the recent literature. Intensive Care Med. 2008;34(1):61-9.

13. Hemptinne Q, Remmelink M, Brimioulle S, Salmon I, Vincent JL. ARDS: A clinicopathological confrontation. Chest. 2009;135(4):944-49.

14. Iribarren C, Jacobs DR Jr, Sidney S, Gross MD, Eisner MD. Cigarette smoking, alcohol consumption, and risk of ARDS: a 15-year cohort study in a managed care setting. Chest. 2000;117(1):163-8.

15. Bajpai S, Bichile L. Mortality analysis of patients of acute febrile illness during monsoon in a tertiary care hospital of Mumbai. Infect Dis Clin Pract. 2008;16:294-7.

16. Lu Y, Song Z, Zhau X, Huang S, Zhu D, Yang C Bai X et al. A 12- month clinical survey of incidence and outcome of acute respiratory distress syndrome in Shanghai intensive care units. Intensive Care Med. 2004;30(12):2197-203.

17. Meduri GU, Golden E, Freire AX, Taylor E, Zaman M, Carson SJ. Methylprednisolone infusion in early severe ARDS: Results of a randomized controlled trial. Chest. 2007;131(4):954-63.

18. Steinberg KP, Hudson LD, Goodman RB, Hough CL, Lanken PN, Hyzy R, et al. ARDS Network. Efficacy and Safety of Corticosteroids for Persistent Acute Respiratory Distress Syndrome. N Engl J Med. 2006;354(16):1671-84.

19. Peter JV, John P, Graham PL, Moran JL, George IA, Bersten A. Corticosteroids in the prevention and treatment of acute respiratory distress syndrome(ARDS) in adults: meta-analysis. BMJ. 2008:336(7651):1006-9.

20. Awasthi S, Prabhu V, Francis JM, Kunhikatta V, Thunga G. Corticosteroid an ambiguity in ARDS. JPPR. 2013;43(3):248-9.

Article History: Submission Date: 04-06-16; Revision Date: 30-07-16; Accepted Date: 27-08-16.

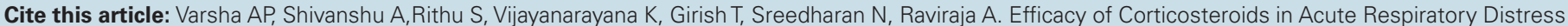
Syndrome: An Observational Study. J Young Pharm. 2017;9(1):65-8. 